\title{
Palindromic Symmetry in Genomes and Evolution ${ }^{\dagger}$
}

\author{
Michael Sadovsky ${ }^{1, *}$ and Yaroslav Grebnev ${ }^{2}$ \\ 1 Institute of Computational Modelling of SB RAS, 660036 Krasnoyarsk, Russia \\ 2 Institute of Fundamental Biology and Biotechnology, Siberian Federal University, Svobodny Prospect, 79, \\ 660041 Krasnoyarsk, Russia; yaroslav.grebnev@gmail.com \\ * Correspondence: msad@icm.krasn.ru \\ + Presented at Symmetry 2017-The First International Conference on Symmetry, Barcelona, Spain, \\ 16-18 October 2017.
}

Published: 12 January 2018

Any genome is a symbol sequence from a four-letter alphabet. A lot can be retrieved from the studies of frequency distribution in ensembles of considerably short strings, of a given length. A palindrome is two strings that read equally in opposite directions; a complementary palindrome is two strings that read equally in opposite directions with respect to Chargaff's substitution rule (A $\leftrightarrow$ $\mathrm{T}$ and $\mathrm{C} \leftrightarrow \mathrm{G}$ ). The symmetry observed over a genome manifests in a proximal equivalence of the strings comprising a complementary palindrome; it should be stressed that the frequencies are counted over a single strand. Thus, it seems that a duality exists between the symmetry and the double helix structure. The frequency equality is not exact, but shows some deviations. We studied a number of genomes of various taxa to determine the deviation figures in order to learn the relation between the deviation figure, and taxonomy of a genome bearer. The deviation figures have been studied for the strings that range from 1 to 8 in length. Also, a theoretical estimation of the deviation figures has been obtained, for various models of a genetic sequence. A dependence of deviation figures from taxonomy has been observed for high taxa ranks, only. Besides, an inhomogeneity of a genome in terms of the figures has been studied for a number of organisms. A new inner structuredness manifesting in divergence of the deviation figures observed for various genome regions was found in all the genomes.

Conflicts of Interest: The authors declare no conflict of interest.

(C) 2018 by the authors. Licensee MDPI, Basel, Switzerland. This article is an open access article distributed under the terms and conditions of the Creative Commons Attribution (CC BY) license (http://creativecommons.org/licenses/by/4.0/). 